\title{
Review Paper on Shallow Learning and Deep Learning Methods for Network security
}

\author{
Afzal Ahmad ${ }^{1 *}$, Mohammad Asif ${ }^{2}$, Shaikh Rohan Ali ${ }^{3}$ \\ ${ }^{1}$ Computer Dept. Jamia Polytechnic, MSBTE, Mumbai, India \\ ${ }^{2}$ Computer Department, Jamia Institute of Engineering \& Management Studies, N.M.U., Jalgaon, India \\ ${ }^{3}$ Computer Department, Jamia Institute of Engineering \& Management Studies, N.M.U., Jalgaon, India
}

Available online at: www.isroset.org

Received: 15/Oct/2018, Accepted: 24/Oct/ 2018, Online: 31/Oct/2018

\begin{abstract}
Machine learning is embraced in an extensive variety of areas where it demonstrates its predominance over customary lead based calculations. These strategies are being coordinated in digital recognition frameworks with the objective of supporting or notwithstanding supplanting the principal level of security experts although the total mechanization of identification and examination is a luring objective, the adequacy of machine learning in digital security must be assessed with the due steadiness. With the improvement of the Internet, digital assaults are changing quickly and the digital security circumstance isn't hopeful. Since information are so critical in ML/DL strategies, we portray a portion of the normally utilized system datasets utilized in ML/DL, examine the difficulties of utilizing ML/DL for digital security and give recommendations to look into bearings. Malware has developed over the previous decades including novel engendering vectors, strong versatility methods and also different and progressively propelled assault procedures. The most recent manifestation of malware is the infamous bot malware that furnish the aggressor with the capacity to remotely control traded off machines therefore making them a piece of systems of bargained machines otherwise called botnets. Bot malware depend on the Internet for proliferation, speaking with the remote assailant and executing assorted noxious exercises. As system movement action is one of the principle characteristics of malware and botnet task, activity investigation is frequently observed as one of the key methods for recognizing traded off machines inside the system. We present an examination, routed to security experts, of machine learning methods connected to the recognition of interruption, malware, and spam.
\end{abstract}

Keywords - Machine learning, Deep learning, Cyber security, Adversarial learning

\section{INTRODUCTION}

With the inexorably inside and out coordination of the Internet and social life, the Internet is changing how individuals learn and function, however it additionally opens us to progressively genuine security dangers. Step by step instructions to distinguish different system assaults, especially not beforehand observed assaults, is a key issue to be settled earnestly. Digital security is an arrangement of advances and procedures intended to ensure PCs, systems, projects and information from assaults and unapproved access, adjustment, or decimation [1]. The interest and inescapability of machine learning (ML) is developing. Existing techniques are being enhanced, and their capacity to comprehend and answer main problems is very valued. These accomplishments have prompted the appropriation of machine learning in a few areas, for example, PC vision, therapeutic examination, gaming and web based life promoting [2]. In a few situations, machine learning methods speak to the best decision over conventional run based calculations and even human administrators [3]. This pattern is additionally influencing the digital security field where some discovery frameworks are being redesigned with ML parts [4]. Our investigation depends on a broad survey of the writing and on unique examinations performed on genuine, vast endeavors and system movement. Other scholastic papers think about ML answers for digital security by thinking about one particular application (e.g.: [4], [3], [5]) and are commonly situated to Artificial Intelligence (AI) specialists as opposed to security administrators. we present a unique scientific classification of machine learning digital security approaches. At that point, we delineate distinguished classes of calculations to three issues where machine learning is as of now connected: interruption identification, malware examination, spam and phishing recognition. At long last, we dissect the principle restrictions of existing methodologies. Our examination features advantages and disadvantages of various strategies, particularly as far as false positive or false negative alerts.

\section{LIKENESS AND DIFFERENCES IN ML AND DL}

There are numerous riddles about the relationship among ML, DL, and man-made consciousness (AI). AI is another innovative science that reviews and creates speculations, strategies, methods, and applications that recreate, grow and broaden human insight [6].

It is a part of software engineering that tries to comprehend the embodiment of knowledge and to deliver another kind 
of canny machine that reacts in a way like human insight. Research around there incorporates apply autonomy, PC vision, nature dialect handling and master frameworks. AI can reenact the data procedure of human awareness, considering. AI isn't human insight, however taking on a similar mindset as a human may likewise surpass human knowledge. ML is a part of AI and is firmly identified with (and frequently covers with) computational insights, which likewise centers around forecast making utilizing PCs. It has solid connections to scientific streamlining, which conveys strategies, hypothesis and application spaces to the field. ML is at times conflated with information mining [7], yet the last subfield concentrates more on exploratory information examination and is known as unsupervised learning. ML can likewise be unsupervised and be utilized to learn and set up pattern conduct proles for different elements and afterward used to discover important peculiarities [8]. The pioneer of ML, Arthur Samuel, characterized ML as a "field of concentrate that enables PCs to learn without being unequivocally customized." ML basically centers around order and relapse in light of known highlights beforehand gained from the preparation information. DL is another field in machine-learning research. Its inspiration lies in the foundation of a neural system that reenacts the human cerebrum for explanatory learning. It emulates the human cerebrum system to decipher information, for example, pictures, sounds and messages [9].An ML method primarily includes the following four steps [7]:

- Feature Engineering. Choice as a basis for prediction (attributes, features).

- Choose the appropriate machine learning algorithm. (Such as classification algorithm or regression algorithm, high complexity or fast)

- Train and evaluate model performance. (For different algorithms, evaluate and select the best performing model.)

- Use the trained model to classify or predict the unknown data.

\section{LITURATURE SURVEY}

Inspiration for this theme originates from the huge effect information preprocessing has on the exactness and ability of ML-based indicators. The survey of information preprocessing finds that numerous locators confine their perspective of system movement to the TCP/IP bundle headers. Time-based insights can be gotten from these headers to distinguish organize examines, arrange worm conduct, and DoS assaults. Various different finders perform further investigation of demand parcels to distinguish assaults against system administrations and system applications. Later methodologies examinations full administration reactions to identify assaults focusing on customers. The audit covers an extensive variety of identifiers, featuring which classes of assault are perceivable by every one of these methodologies. Data preprocessing is found to overwhelmingly depend on master area learning for recognizing the most applicable parts of system activity and for developing the underlying competitor set of movement highlights. Then again, robotized strategies have been broadly utilized for highlight extraction to decrease information dimensionality, and highlight choice to locate the most pertinent subset of highlights from this hopeful set. The survey demonstrates a pattern towards more profound parcel examination to develop more important highlights through focused substance parsing. These setting touchy highlights are suited to recognizing current assaults at the application layer.

Network Intrusion Detection Systems: -NIDS screen PC systems for indications of trade off, or endeavored bargain. In actuality, they characterize each movement perception as vindictive or not. They can be intended to either perform abuse discovery or inconsistency location. Abuse based NIDS recognize known malevolent action, while irregularity based distinguish unordinary movement. Misuse built NIDS for the most part depends concerning marks made by territory authorities. Famous open-source executions of this compose are grunt [10] and brother [11]. Business NIDS are likewise by and large abuse based in light of the fact that, similarly as with AV programming, low false positive rates can be accomplished. These frameworks require general mark updates to identify the most recent assaults. In any case, given the regularly expanding rundown of malware, the activity of continually breaking down and making marks is work serious. Adding to the trouble is the prepared accessibility of toolboxes from the Web which enable aggressors to make new malware. The toolboxes likewise enable endeavors to be repackaged into one of a kind malware examples utilizing polymorphism. This has prompted theory that mark based $\mathrm{AV}$ and interruption location is unsustainable [12]. Abuse based frameworks are additionally for the most part unfit to identify novel or zero-day assaults [14].

Indeed, even a $1 \%$ false positive rate results in countless alarms when kept running on the vast volumes of movement normal in current systems. This is known as the base rate deception [13]. Peculiarity based methodologies are as yet a functioning zone of research. This writing audit covers more abnormality identifiers than abuse finders 
both in light of the fact that oddity indicators are all the more effectively being inquired about, and on the grounds that they for the most part utilize ML (to display typical conduct).

Most audits of oddity constructed NIDS in this way gather in light of their center calculations. This survey rather covers their information preprocessing systems, concentrating on what parts of the system movement are examined, and what include development and determination strategies have been utilized. The survey examinations applicable inconsistency based NIDS productions from the most recent decade. The center is inspired by the way that information preprocessing requires a lot of exertion, a specifically impacts on the precision and capacity of the downstream calculation, [16, 15]. In this way, information preprocessing shapes a basic piece of inconsistency based NIDS. The center is likewise propelled by the way that substance based assaults have turned out to be more applicable, while more established DoS, arrange test and system worm assaults have to a great extent been moderated by border barriers. This survey likewise takes note of any procedures which have been connected to genuine world systems. Adapting to true information infers considering computational many-sided quality, equipment assets, and contrasts between preparing information and genuine data.

1. It exhaustively audits the highlights got from system activity, and the related information preprocessing methods which have been utilized in inconsistency based NIDS since 1999. These parts of NIDS are generally imperative since they decide, to a huge degree, its recognition inclusion.

2. It bunches inconsistency based NIDS by the sorts of system activity highlights utilized for location. The point is to indicate where the larger part of research has been engaged. The gatherings demonstrate a pattern from beforehand utilizing parcel header includes solely, to utilizing more payload highlights.

Likewise precluded are papers exclusively tending to NIDS execution, for example, utilizing equipment quickening or parallel designs. While execution is an imperative viewpoint for NIDS checking high transfer speed joins, it is a territory deserving of a different report. The audit endeavors to cover a wide assortment of system interruptions instead of simply the conventional test and DoS assaults. In any case, a prominent exclusion is botnet location, again to confine scope.

Figure 1 graphs the numbers of reviewed papers using each of the identified feature types. It shows the largest group of papers use features derived only from network packet headers. It also shows that a significant number of papers depend on the features in the KDD Cup 99 dataset. While a number of reviewed papers use features derived from packet contents or payloads, most of those analyses the payloads of requests to servers. This literature reviews first covers packet header features.

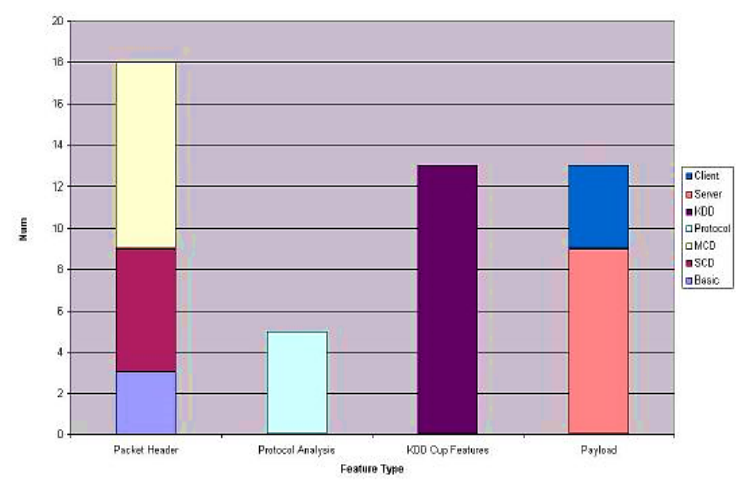

\section{Fig1: Number of published anomaly-based NIDS papers vs. feature type used}

\section{METHODOLOGY}

For experimentation, content ordering strategies were utilized for parsing the messages. All connections were evacuated, "header data everything being equal and html labels" from the messages' bodies and also their particular components were removed. A short time later, a stemming calculation was connected and all the unimportant words were expelled. At last, all things were arranged by their recurrence in messages. Because of this work, it tends to be reasoned that LR is a more ideal alternative among clients because of low false positive rate (ordinarily, clients would not need their real messages to be misclassified as garbage). Likewise, LR has the most astounding exactness and moderately high review in correlation with different classifiers under thought. The examination of accuracy, review, and F-measure is given in Table 1.

Table 1: Comparison of precision, recall, and F1 [1]

\begin{tabular}{|c|c|c|c|}
\hline Classifier & Precision & Recall & F1 \\
\hline LR & $\mathbf{9 5 . 1 1} \%$ & $\mathbf{8 2 . 9 6} \%$ & $\mathbf{8 8 . 5 9} \%$ \\
\hline CART & $\mathbf{9 2 . 3 2} \%$ & $\mathbf{8 7 . 0 7 \%}$ & $\mathbf{8 9 . 5 9 \%}$ \\
\hline SVM & $\mathbf{9 2 . 0 8 \%}$ & $\mathbf{8 2 . 7 4} \%$ & $\mathbf{8 7 . 0 7} \%$ \\
\hline NNet & $\mathbf{9 4 . 1 5} \%$ & $\mathbf{7 8 . 2 8} \%$ & $\mathbf{8 5 . 4 5} \%$ \\
\hline BART & $\mathbf{9 4 . 1 8} \%$ & $\mathbf{8 1 . 0 8 \%}$ & $\mathbf{8 7 . 0 9} \%$ \\
\hline RF & $\mathbf{9 1 . 7 1} \%$ & $\mathbf{8 8 . 8 8} \%$ & $\mathbf{9 0 . 2 4} \%$ \\
\hline
\end{tabular}

Machine Learning Algorithms for Cyber Security: Machine learning incorporates a vast assortment of ideal models in constant advancement, displaying feeble limits and cross connections. Besides, unique perspectives and applications may prompt distinctive arrangements. The principal separate prove in Figure 1 is between the 
customary ML calculations, which today can be alluded to as Shallow Learning (SL), contrary to the later Deep Learning (DL). Shallow Learning requires an area master (that is, a component design) who can play out the basic assignment of distinguishing the important information qualities previously executing the SL calculation. Profound Learning depends on a multi-layered portrayal of the info information and can perform highlight choice self-ruling through a procedure characterized portrayal learning. SL and DL methodologies can be additionally portrayed by recognizing directed and unsupervised calculations. The previous systems require a preparation procedure with a substantial and delegate set of information that have been already grouped by a human master or through different means. The last methodologies don't require a pre-named preparing dataset. In this segment, we consider and look at the most famous classifications of ML calculations, which show up as the leaves of the order tree in Figure 2.

Here we discuss algorithm one by one which are most useful Shallow Learning

1) Supervised SL algorithms

- $\quad$ Naïve Bayes (NB).

- Logistic Regression (LR).

- Support Vector Machines (SVM).

- Random Forest (RF).

- Hidden Markov Models (HMM).

- K-Nearest Neighbor (KNN).

- Shallow Neural Network (SNN).

2) Unsupervised SL algorithms

- Clustering.

- Association.

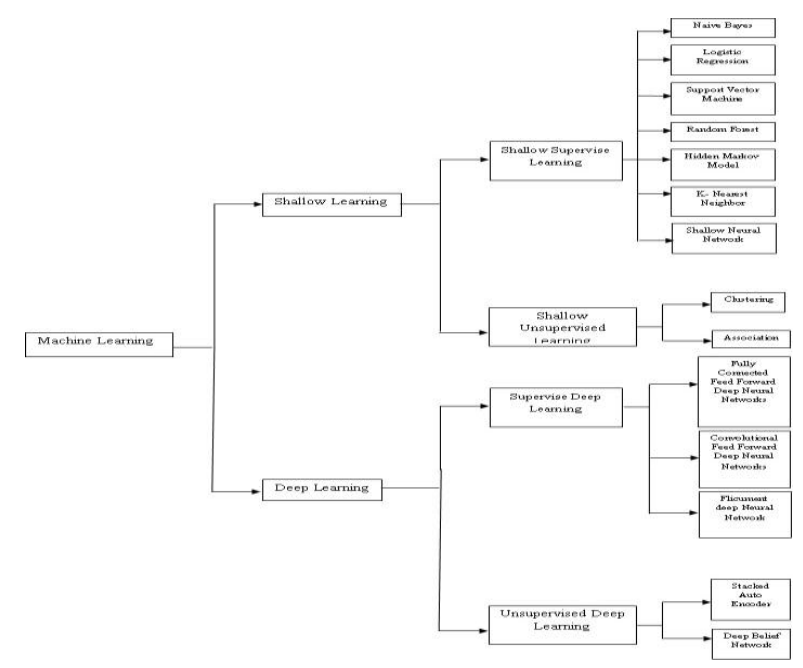

FIGURE2. CLASSIFICATION OF ML ALGORITHMS FOR CYBER SECURITY APPLICATIONS

What is Naive Bayes algorithm?
It is a course of action strategy in perspective of Bayes' Theorem with an assumption of independence among markers. In direct terms, a Naive Bayes classifier acknowledge that the proximity of a particular component in a class is detached to the closeness of some other component. For example, a characteristic item may be seen as an apple in case it is red, round, and around 3 sneaks in broadness. Despite whether these features depend upon each other or upon the nearness of substitute features, these properties openly add to the probability that this common item is an apple and that is the reason it is known as 'Naive'. [17]

Bayes theorem gives a method for ascertaining back likelihood as probability $\mathrm{P}(\mathrm{c} \mid \mathrm{x})$ from $\mathrm{P}(\mathrm{c}), \mathrm{P}(\mathrm{x})$ and $\mathrm{P}(\mathrm{x} \mid \mathrm{c})$. Take a gander at the condition underneath: [17]

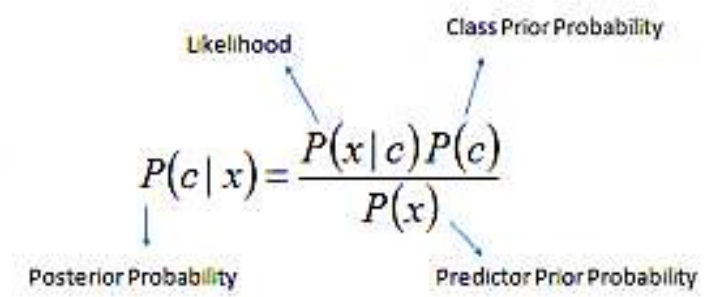

$$
P(c \mid \mathrm{X})=P\left(x_{1} \mid c\right) \times P\left(x_{2} \mid c\right) \times \cdots \times P\left(x_{n} \mid c\right) \times P(c)
$$

Above,

- $\quad \mathrm{P}(\mathrm{c} \mid \mathrm{x})$ is the posterior probability of class (c, target) given predictor ( $\mathrm{x}$, attributes).

- $\mathrm{P}(\mathrm{c})$ is the prior probability of class.

- $\mathrm{P}(\mathrm{x} \mid \mathrm{c})$ is the likelihood which is the probability of predictor given class.

- $\mathrm{P}(\mathrm{x})$ is the prior probability of predictor.

KNN algorithm: -KNN can be utilized for both order and relapse prescient issues. Notwithstanding, it is all the more generally utilized in arrangement issues in the business. To assess any system, we for the most part take a gander at 3 critical viewpoints: [18]

1. Ease to interpret output

2. Calculation time

3. Predictive Power

\begin{tabular}{|l|r|r|r|r|r|}
\cline { 2 - 5 } \multicolumn{1}{c|}{} & Logistic Regression & CART & Random Forest & KNN \\
\hline 1. Ease to interpret output & 2 & 3 & 1 & 3 \\
\hline 2. Calculation time & 3 & 2 & 1 & 3 \\
\hline 3. Predictive Power & 2 & 2 & 3 & 2 \\
\hline
\end{tabular}


input : Input Poisson Spike Train spikes, Number of Time-Steps \#timesteps[19]

output: Weight-normalization / Threshold-balancing factors vth,norm[i] for each neural layer

(net.layer[i]) of the network net

1 initialization vth,norm $[\mathrm{i}]=0 \forall \mathrm{i}=1, \ldots$, \#net.layer;

$2 / /$ Set input of 1 st layer equal to spike train

3 net.layer[1].input $=$ spikes;

4 for $\mathrm{i} \leftarrow 1$ to \#net.layer do

5 for $\mathrm{t} \leftarrow 1$ to \#timesteps do

6 // Forward pass spike-train for neuron layer-i characterized by membrane

potential net.layer[i].vmem and threshold net.layer[i].vth

7 net.layer[i] : forward(net.layer[i].input) ;

8 // Determine Threshold-balancing factor according to maximum SNN activation,

net.layer[i].vmem.input

$9 v$ th,norm[i]=max $(v$ th, norm[i], $\max ($ net.layer[i].vmem.inpu

t));

10 end

$11 / /$ Threshold-balance layer-i

12 net.layer[i].vth = vth,norm[i];

13 // Record input spike-train for next layer

14 net.layer[i + 1].input $=$ net.layer[i] : forward(net.layer[i].input);

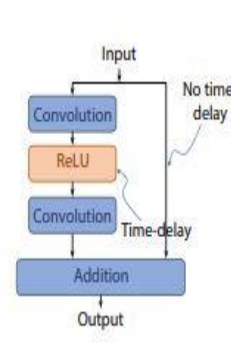

(a)

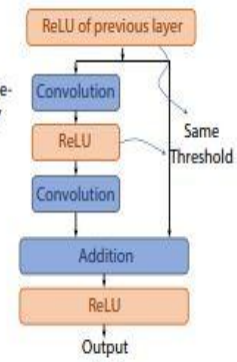

(b)

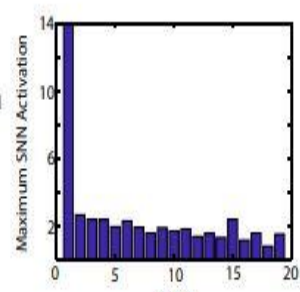

(c)
Figure 3: (a) The basic ResNet functional unit, (b) Design constraints introduced in the functional unit to ensure nearlossless ANN-SNN conversion, (c) Typical maximum SNN activations for a ResNet having junction ReLU layers but the non-identity and identity input paths not having the same spiking threshold. While this is not representative of the case with equal thresholds in the two paths, it does justify the claim that after a few initial layers, the maximum SNN activations decay to values close to unity due to the identity mapping.

\section{Unsupervised SL algorithms: -}

Clustering algorithm: -Clustering can be considered the most important unsupervised learning problem; so, as every other problem of this kind, it deals with finding a structure in a collection of unlabeled data. A loose definition of clustering could be "the process of organizing objects into groups whose members are similar in some way". A cluster is therefore a collection of objects which are "similar" between them and are "dissimilar" to the objects belonging to other clusters. We can show this with a simple graphical example

\section{Unsupervised SL algorithms: -}

Clustering: -In the primary case information are assembled in a selective way, so that if a specific datum has a place with a clear bunch then it couldn't be incorporated into another group. A basic case of that is appeared in the figure underneath, where the partition of focuses is accomplished by a straight line on a bidimensional plane. Despite what might be expected the second kind, the covering grouping, utilizes fuzzy sets to bunch information, so each point may have a place with at least two bunches with various degrees of participation. For this situation, information will be related to a proper enrollment esteem [21].

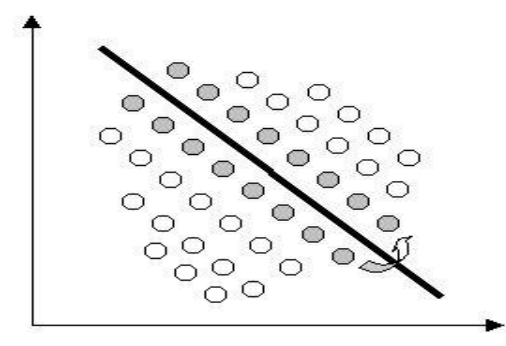

Rather, a various leveled bunching calculation depends on the joining between the two closest groups. The starting condition is acknowledged by setting each datum as a cluster. After a couple of emphasis, it achieves the last groups needed. At last, the last sort of bunching utilizes a totally probabilistic methodology.

Association: -Algorithm: - Rule generation apriori algorithm [22].

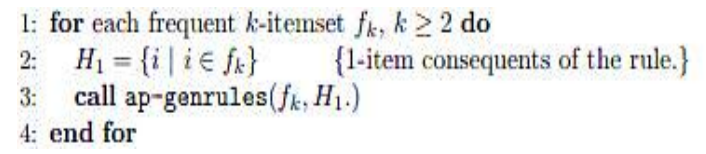

Algorithm: - procedure ap-genrules $\left(\mathrm{f}_{\mathrm{k}}, \mathrm{H}_{\mathrm{m}}\right)$ 
1: $k=\left|f_{k}\right| \quad$ \{size of frequent itemset.\}

2: $m=\left|H_{m}\right| \quad$ \{size of rule consequent.\}

3: if $k>m+1$ then

$H_{m+1}=$ apriori-gen $\left(H_{m}\right)$.

for each $h_{m+1} \in H_{m+1}$ do

$\operatorname{conf}=\sigma\left(f_{k}\right) / \sigma\left(f_{k}-h_{m+1}\right)$.

if conf $\geq \operatorname{minconf}$ then

output the rule $\left(f_{k}-h_{m+1}\right) \longrightarrow h_{m+1}$.

else

delete $h_{m+1}$ from $H_{m+1}$.

end if

end for

call ap-genrules $\left(f_{k}, H_{m+1}\right)$

: end if

\section{Deep Learning:}

All DL algorithms are primarily based on Deep Neural Networks (DNN), which can be big neural networks organized in many layers able to selfsufficient representation learning.

Fully-connected Feedforward Deep Neural Networks(FNN): -Feedforward neural networks are in most cases used for supervised gaining knowledge of in instances where the data to be learned is neither sequential nor time-based. that is, feedforward neural networks compute a function $f$ on fixed size input xsuch that $f(x)=y$ for training pairs $(x, y)$.on the other hand, recurrent neural networks examine sequential data, computing $g$ on variable length input $X_{k}=\left\{x_{1}, \ldots \ldots \ldots \ldots, x_{k}\right\}$,such that $\mathrm{g}\left(\mathrm{X}_{\mathrm{k}}\right)=\mathrm{y}_{\mathrm{k}}$ or training pairs $\left(\mathrm{x}_{\mathrm{n}}, \mathrm{y}_{\mathrm{n}}\right)$ for all $1 \leq \mathrm{k} \leq \mathrm{n}$.

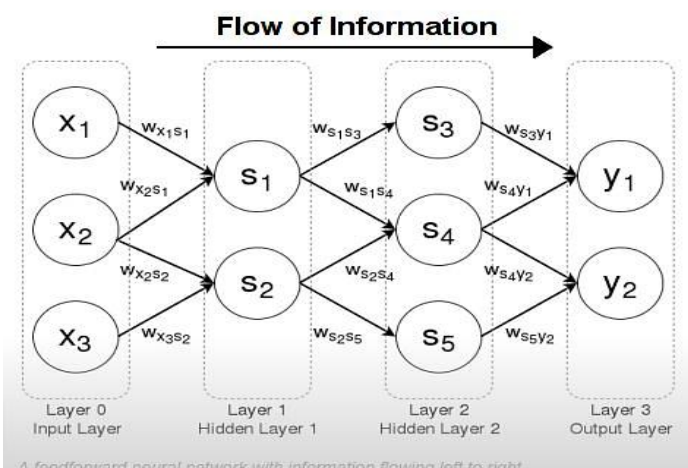

Fig 4:Fully-connected Feedforward Deep Neural Networks

\section{Convolutional Feedforward Deep Neural Networks (CNN):}

Mathematically, the operation is defined as

$$
h_{i j}^{k}=f\left(\left(W^{k} * X\right)_{i j}+b_{k}\right)
$$

where $W^{k}$ is a filter, * is the convolution operator, and $f$ is a nonlinearity

Usually a number of filters $\left\{W^{k}\right\}_{k=1}^{K}$ are applied (each will produce a separate "feature map").

These filters have to be learned

Size of these filters have to be specified

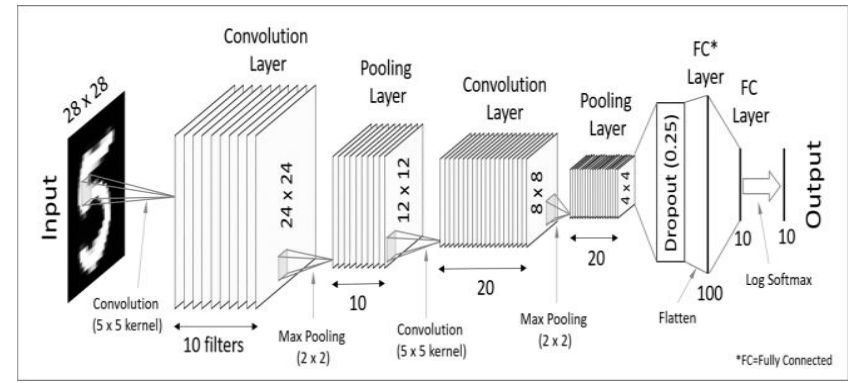

Unsupervised DL algorithms:

Stacked Auto encoders (SAE):As an unmonitored learning set of rules, an auto-encoder community consists of 3 layers: enter layer, hidden layer and output layer [26]. It makes the output layer same to the enter layer, which minimizes the reconstruction mistakes to extract a satisfactory expression of the hidden layer. the autoencoder network based information processing for e-noses includes two steps (as proven in figure 1): first off, the original e-nostril records

$\mid x=\left[x^{(1)} x^{(2)} \cdots x^{(m)}\right]^{T}$

encoded from the input layer to the hidden layer (encoding):

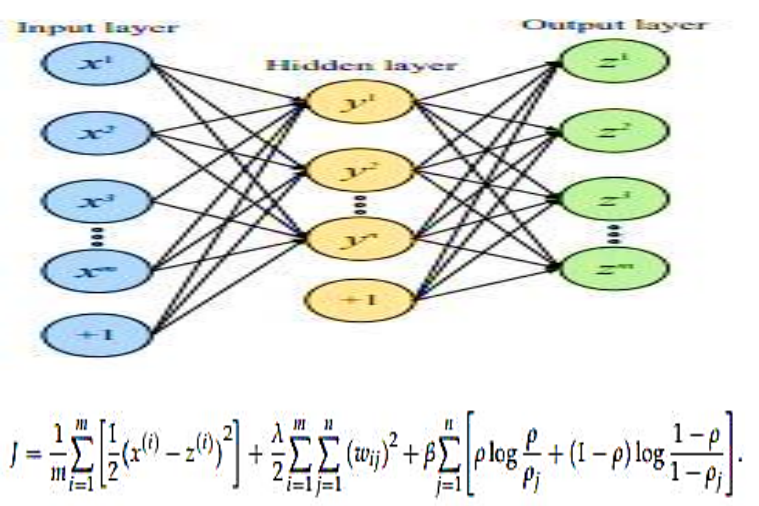

Deep Belief Networks (DBN): As the DBN stacks numerous layers of RBMs, executing a DBN requires preparing each layer of RBM. [23] The hypothetical parts of $\mathrm{RBM}$ and DBN preparing are clarified in the past segment and now we present those calculations in steps. The following calculation demonstrates the means for Contrastive Dissimilarity to prepare RBM [24]. 
TrainUnsupervisedDBN $(\hat{P}, \epsilon, \ell, W, \mathrm{~b}, \mathrm{c}$, mean.field conputation Thain a DBN in a purely unstpervised way, with the greedy layer-wise procedure in which each added layer is trained as an RBM (e.g., by Contrastive Divergence).

$\widehat{P}$ is the input training distribution for the network

$\epsilon$ is a learning rate for the RBM training

$\ell$ is the number of layers to train

$W^{k}$ is the weight matrix for level $k$, for $k$ from 1 to $\ell$

$\mathrm{b}^{k}$ is the visible units offset vector for RBM at level $k$, for $k$ from 1 to $\ell$

$\mathrm{c}^{k}$ is the hidden units offset vector for RBM at level $k$, for $k$ from 1 to $\ell$

mean field computation is a Boolean that is true iff training data at each additional level is obtained by a mean-field approximation instead of stochastic sampling

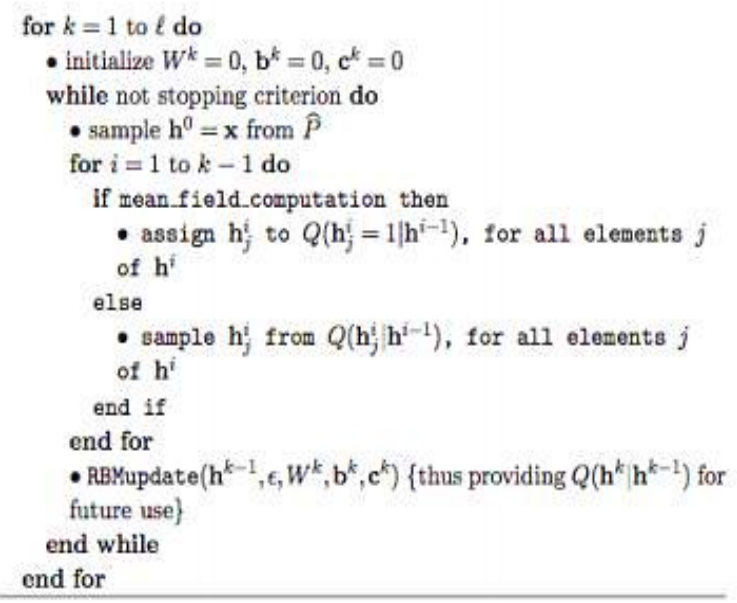

Fig 5: DBN learning algorithm

\section{RESUlTS AND Discussion}

First of all we compare Naïve Bayes with $\mathrm{KNN}$ as performance scenario

Comparative performance behavior: As shown in the parent when nearest neighbor and naïve basin classifier are used suggests an awesome status whilst facts units training is commenced with small wide variety of documents and when the range of documents will increase the distinction starts off evolved showing the overall performance of those classifiers differs. For the larger education sets, the overall performance of naïve basin is lots better than KNN classifier however it does not appear that if capabilities are increasing then the overall performance drops.

Comparative processing time behavior: When processing time is considered it is shown that the processing time is totally depend upon the size of test set as the size increases the processing time increases and remain same for these two classifiers and if different number of documents (and of different test size) are used then we can have observed the processing time differences. As the number of features changes the training time for both classifiers is to train data is required much and as the number of features increases the time required to train data set is less
To go with the spiritualization of clustering and association algorithm go with comparison between association algorithm vs cluster algorithm

- Cluster evaluation or clustering is the assignment of grouping a hard and fast of gadgets in one of these manner that gadgets inside the equal institution (known as a cluster) are more comparable (in a few sense or some other) to every aside from to ones in other corporations (clusters).

- $\quad$ association rule getting to know is a way for coming across thrilling relations between variables in huge databases

Now we go with comparison between FNN and CNN algorithm as the field of deep learning.

\section{FNN database training process}

on this procedure, the fake generic price (a long way), authentic commonplace rate (TAR), fake rejected price (FRR), precision, and accuracy were decided. the proportion of a long way is zero. $0121 \%$. This suggests that during the FNN training technique, handiest 1.32 out of 7044 records images are falsely commonplace. For TAR, 7031.45 out of 7044 statistics images are genuinely popular, and 12.54 out of 7044 facts pix are falsely rejected in FRR. This training process is reliable because of the excessive precision and accuracy charges, which were $99.9802 \%$ and $99.9229 \%$

\section{CNN database training process}

on this technique, the proportion of a ways is zero.032968\%. This suggests that during the CNN database training procedure, 1.76 out of 7044 information pix are falsely time-honored. For TAR, 7044 out of 7044 facts images are truly usual, and 0 out of 7044 statistics photos are falsely rejected in FRR. This CNN database training procedure produces $99.96 \%$ precision rate and $99.9812 \%$ accuracy charge.

there is a sizeable distinction between the preceding strategies' performances and the proposed technique. Lan and Kuo [29] brought a method with $90.31 \%$ as the detected percentage, which means that that five out of 52 check photographs were detected falsely. Peng and Harlow [30] performed $91.00 \%$ accuracy; 90 out of 1000 check photographs were detected falsely. Shaoing et al. [31] offered a method with the detection fee of $93.13 \%$ for automobile type (46 out of 670 of test pics have been detected). The summary of comparisons is defined in table 3 therefore, the mixture of the FNN and CNN strategies has extra credit as compared to the preceding class strategies.

Now we compare unsupervised algorithm as DBN vs SAE algorithm comparing the structure of both a stacked auto encoder (SA) or a stacked de noising auto encoder (SdA) (they're identical) with the form of a deep belief network (DBN), you likely can see that the vital variations consist inside the output layer and the course of the connections between layers. inside the first architecture the output layer 
is emerge as independent from the enter layer however in the 2nd shape it coincides with it. this is additionally remarked through the arrows connecting the layers. In a SA or Sd A the facts flow unidirectional from the enter layer, thru the hidden layer, up to the output layer. In a DBN the records flows both ways some of the visible (input/output) layer and the hidden layer [32].

\section{CONCLUSION AND FUTURE SCOPE}

The machine learning is a very vast field of computer science in modern technology, through the availability of internet. The major issues in this evolutionary world is to communicate data passes data deal with security. The convolutionary deployment of the deep learning technology in cyber security. Now we are concentrate in this paper is some popular algorithm use in this era.

According to our analysis the useable algorithm we splitting up onto two main regions i.e. supervised and unsupervised under each sub domain of shallow learning and deep learning.

To total analysis in shallow learning the impactful algorithm is "Clustering" because it facilities that each point should be classified inside the specific cluster. Cluster evaluation itself isn't always one unique algorithm, but the preferred assignment to be solved. it can be performed by means of various algorithms that range significantly in their expertise of what constitutes a cluster and the way to effectively discover them. popular notions of clusters encompass agencies with small distances among cluster individuals, dense regions of the data area, durations or particular statistical distributions. Clustering can consequently be formulated as a multi-objective optimization hassle. the correct clustering set of rules and parameter settings (which include parameters such as the space feature to apply, a density threshold or the number of anticipated clusters) rely upon the individual records set and supposed use of the results. Cluster evaluation as such is not an automated task, but an iterative manner of understanding discovery or interactive multi-objective optimization that includes trial and failure. it's far regularly important to adjust records preprocessing and model parameters until the result achieves the favored residences.

In case of deep learning scenario DBN have a grate impaction rather than other set of rule i.e. an algorithm. DBNs are actually a more recent sort of community than maximum other community sorts, particularly back propagation networks, and are different in general within the unsupervised education phase and that their output is stochastic. In supervised learning they tend to be skilled with back propagation within the final section. The unsupervised getting to know has a theoretical foundation based on strength in preference to minimizing the reconstruction blunders, which makes them exciting theoretically. In exercise their unsupervised education section appears equivalent in performance and behavior with demising auto encoders. Deep belief Networks
(DBNs) with restrained Boltzmann machine (RBM) for featuring a brand new technique for developing images with better transparency and decrease complexity of running time. [33].

The outcomes of the experiments conducted on this thesis display that a categorized dataset with a proportional set of examples trained with the Deep mastering algorithm can as it should be come across unusual interest. This approach lets in for multiple log source sorts to be aligned the use of a sliding time window and affords a scalable solution which is a far wanted feature.

In an average organization environment, the quantity of $\log$ data processed should range from numerous hundred gigabytes to a terabyte every day. The prototype developed in this studies became incredibly small consisting of a fixed of eighteen functions from 3 exceptional log source kinds totaling about twenty-5 gigabytes in length. This research proven the prototype may want to very appropriately model low complexity records with a shallow community. but, the complexity of the records will increase as extra log assets and capabilities are delivered. This research confirmed that relatively complicated facts might be as it should be modeled the use of a deep neural network.

Detecting a cyber-attack is just the start of a protracted, complex investigative technique. the security analyst may additionally want to carry out threat mitigation actions, along with blacklisting originating source IP's and locking debts. Logs files need to be examined to perceive any compromised accounts, originating IP's, and all sources accessed by using the attacker. All associated activities need to be amassed and tested numerous weeks or even months earlier than the detected occasion. ability regions of future work are automatic correlation and evaluation of the $\log$ records from cyber-attacks. additional gadget learning algorithms and analysis required for automatic correlation can placed a stress on computing resources depending on the extent of information to be searched and speed of the log records being accrued. extra regions of future work encompass constructing a dispensed computing implementation which includes Hadoop with terabytes of $\log$ facts.

-Increase the supported listing of network visitor's protocols: The framework has been tested with HTTP and DNS site visitors in addition to net glide summaries. Similarly, paintings will check help of other network protocols.

- Make bigger framework to aid unsupervised ML: The function choice" step within the framework currently requires labeled datasets to find the most discriminative capabilities. Destiny paintings will put off this framework requirement to guide unsupervised ML with unlabeled data. Big effort on this thesis went into making sure experiments had been representative of real-global situations. This ensured our effects have been significant in modern networks Our datasets were snapshots of community traffic which can be analyzed opine in batch mode. For the detectors to paintings on a stay community, 
several operational elements could need to be addressed. those are mentioned include scalability, generating capabilities on live visitors, integrating more than one detectors for efficiency, and publish-processing indicators. characteristic engineering is a problem while applying ML to any hassle which includes popular fields such optical individual popularity, speech reputation, photograph popularity and language translation. even as function engineering is generally carried out by means of area specialists and is specific to every area, latest advances in deep gaining knowledge of have confirmed a less manual, facts driven technique with parallels to our computerized function engineering method. this is discussed in phase. Similarly work ought to inspect whether deep gaining knowledge of will be leveraged in the automatic function engineering framework.

\section{REFERENCES}

[1] S. Aftergood, "Cybersecurity: The cold war online," Nature, vol. 547,no. 7661, pp. 30_31, Jul. 2017.

[2] M. I. Jordan and T. M. Mitchell, "Machine learning: Trends, perspectives, and prospects," Science, 2015.

[3] A. Buczak and E. Guven, "A survey of data mining and machine learning methods for cyber security intrusion detection," IEEE Communications Surveys \& Tutorials, 2015.

[4] E. Blanzieri and A. Bryl, "A survey of learning-based techniques of email spam filtering," Artificial Intelligence Review, 2008.

[5] J. Gardiner and S. Nagaraja, "On the Security of Machine Learning in Malware C8C Detection," ACM Computing Surveys, 2016.

[6] R. G. Smith and J. Eckroth, "Building AI applications: Yesterday, today, and tomorrow," AI Mag., vol. 31, no. 1, pp. 6_22, 2017.

[7] P. Louridas and C. Ebert, "Machine learning," IEEE Softw., vol. 33, no. 5,pp. 110115, Sep./Oct. 2016.

[8] M. I. Jordan and T. M. Mitchell, "Machine learning: Trends, perspectives, and prospects," Science, vol. 349, no. 6245, pp. 255260, 2015.

[9] Y. LeCun, Y. Bengio, and G. Hinton, “Deep learning," Nature, vol. 521,pp. 429444, May 2015.

[10] M. Roesch. Snort-lightweight intrusion detection for networks. In Proceedingsof the 13th USENIX Conference on System Administration, pages229-231. Seattle, Washington, 1999.

[11] M. Vallentin, R. Sommer, J. Lee, C. Leres, V. Paxson, and B. Tierney. Thenids cluster: Scalable, stateful network intrusion detection on commodityhardware. Lecture Notes in Computer Science, 4630:107-126, 2007.

[12] S. Zanero and S. Savaresi. Unsupervised learning techniques for an intrusion detection system. In SAC '04: Proceedings of the 2004 ACM symposium on Applied computing, pages 412-419, New York, NY, USA, 2004. ACM. ISBN 1-58113812-1. doi: http://doi:acm:org/10:1145/967900:967988.

[13] S. Axelsson. The base-rate fallacy and the difficulty of intrusion detection. ACM Transactions on Information and System Security (TISSEC), 3(3),2000.
[14] R. Bace and P. Mell. Intrusion detection systems. Technical Report 800-31, National Institute of Standards and Technology (NIST), Special Publication,2001.

[15] S.B. Kotsiantis, D. Kanellopoulos, and P.E. Pintelas. Data preprocessing for supervised learning. International Journal of Computer Science, 1(2): 111-117, 2006.

[16] W. Lee and S.J. Stolfo. A framework for constructing features and models for intrusion detection systems. ACM Transactions on Information and System Security (TISSEC), 3(4):227-261, 2000.

[12] 6 Easy Steps to Learn Naive Bayes Algorithm (with codes in Python and R)SUNIL RAY, SEPTEMBER 11, 2017 https://www.analyticsvidhya.com/blog/2017/09/naive-bayesexplained/

[13] Introduction to k-Nearest Neighbors: Simplified (with implementation in Python)TAVISH SRIVASTAVA, MARCH 26, 2018 https://www.analyticsvidhya.com/blog/2018/03/introductionk-neighbours-algorithm-clustering/

[14] Going Deeper in Spiking Neural Networks: VGG and Residual Architectures AbhronilSenguptaa,*, YutingYeb , Robert Wangb , ChiaoLiub , Kaushik Roya aPurdue University, West Lafayette, IN, USA bFacebook Reality Labs, Redmond, WA, USA

[15]An Introduction to Clustering and different methods of clustering SAURAV KAUSHIK, NOVEMBER 3, 2016

[16] A Tutorial on Clustering Algorithms https://home.deib.polimi.it/matteucc/Clustering/tutorial_html/ kmeans.html

[17] Association analysis: Basic concept and algorithm page no 25-26;

[18] Deep Learning: Feedforward Neural Nets and Convolutional Neural Nets Piyush Rai Machine Learning (CS771A) Nov 2, 2016

[19] Deep Learning website. www.deeplearning.net

[20] Classification with Deep Belief Networks HussamHebbo Jae Won Kim page no:20

[23] Shin, H.C.; Orton, M.R.; Collins, D.J.; Doran, S.J.; Leach, M.O. Stacked Autoencoders for Unsupervised Feature Learning and Multiple Organ Detection in a Pilot Study Using 4D Patient Data. IEEE Trans. Pattern Anal. Mach. Intell. 2013, 35, 1930-1943. [CrossRef] [PubMed]

[25] 25. Sun, W.; Shao, S.; Zhao, R.; Yan, R.; Zhang, X.; Chen, $X$. A sparse auto-encoder-based deep neural network approach for induction motor faults classification. Measurement 2016, 89, 171-178. [CrossRef]

[26] Comparison of Naive Basian and K-NN Classifier Deepak Kanojia Me (Cse) Tieit, Bhopal MahakMotwani Assistant Professor (Cse Department) Tieit, Bhopal Page no 46

[29] L.W. Lan, A.Y. Kuo, "Development of a fuzzy neural network colour image vehicular detection (FNNCIVD) system", IEEE 5th International Conference on Intelligent Transportation System, pp. 88-93, 2002.

[30] S. Peng, C.A. Harlow, "A system for vehicle classification from range imagery", Proceedings of the IEEE 28th Southeastern Symposium on System Theory, pp. 327-331, 1996.

[31] M. Shaoqing, L. Zhengguang, J. Zhang, "Real-time vehicle classification method for multi lanes roads", ICIEA 2009, pp. 960-964, 2009. 
[32] A study on the similarities of Deep Belief Networks and Stacked Autoencoders ANDREA DE GIORGIO page no: 70-79

[33] AN APPLICATION OF DEEP BELIEF NETWORKS FOR 3-DIMENSIONAL IMAGE RECONSTRUCTION 1AMIN EMAMZADEH ESMAEILI NEJAD Engineering \& Computer Science Department, Shiraz University, Shiraz, Iran page no: -8

\section{Authors Profile}

Afzal Ahmad received his Master degree from JNTU Hyderabad India. Currently working as Head of Computer Department \& Assistant Professor in Jamia Polytechnic Akkalkuwa.

Mohammad Asif received his B.E. degreefrom NMU JALGAON INDIA. Currently pursuing M.Tech.in Computer Engineering from BATU Lonere. Working as Lecturer in Computer Department JIEMS Akkalkuwa.

Shaikh Rohan Ali pursuing Bachelor of Engineering from Jamia Institute of Engineering \& Management Studies Akkalkuwa Affiliated to NMU Jalgaon India, Approved by AICTE, New Delhi \& DTE

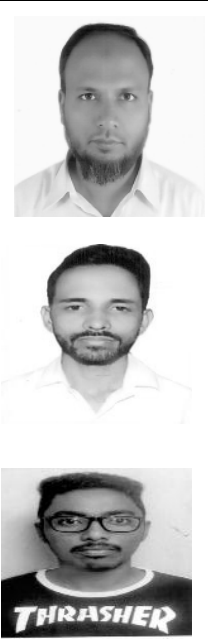
Mumbai. 